\title{
Micronúcleos y otras anormalidades nucleares en el epitelio oral de mujeres expuestas ocupacionalmente a plaguicidas
}

\author{
Rocío Castro $^{1}$, Vanessa Ramírez ${ }^{1,3}$ \& Patricia Cuenca ${ }^{1,2} *$ \\ 1 Instituto de Investigaciones en Salud, Universidad de Costa Rica. \\ 2 Escuela de Biología, Universidad de Costa Rica. \\ 3 Escuela de Nutrición, Universidad de Costa Rica. \\ * Correspondencia: Patricia Cuenca, Ciudad Universitaria Rodrigo Facio, San José Costa Rica, Tel: 506 2243668; FAX \\ 506 2075130; pcuenca@cariari.ucr.ac.cr
}

Recibido 09-XII-2003. Corregido 23-VII-2004. Aceptado 26-VIII-2004.

\begin{abstract}
Micronuclei and other nuclear abnormalities in the oral epithelium of female workers exposed to pesticides. In order to study if banana fields labour exposure to pesticides produces some kind of DNA damage, we determine the presence of micronuclei in epithelial oral cells in working women in Guapiles and Siquirres, Costa Rica, as an effect biomarker. We also analyzed other abnormalities in the nucleus of those cells such as broken-egg, karyolysis or kariorrhexis, to see if there was some kind of genotoxicity or citotoxicity. The women group exposed to pesticides worked in packing bananas plant from different independent farms. The control group of women had never done any farming tasks; they did not live in the banana fields, neither their husband. We got information about the life style, medical and familial history of the participants through an interview. We did not found any significant increment in the frequency of micronuclei form the exposed group compared with the controls. The other nuclei abnormalities showed signs of citotoxicity or genotoxicity in the controls, associated with the intake of coffee and dental x-rays. These results do not rule out at that pesticides used in packing bananas are agents capable of producing damage to the DNA, but it seems that micronuclei from the oral epithelium is not the most adequate marker to measure it. Rev. Biol. Trop. 52(3): 611-621. Epub 2004 Dic 15.
\end{abstract}

Key words: Pesticides, biomonitoring, Costa Rica, banana plantations, female farmers, bucal micronucleous.

Palabras clave: Plaguicidas, monitoreo biológico, cultivo de banano, agricultoras, micronúcleos, mucosa oral, Costa Rica.

Los plaguicidas se han asociado con efectos cancerígenos, teratógenos, mutagénicos, espermatogénicos, fetotóxicos, neurotóxicos o una combinación de varios de estos, tanto en seres humanos como en otras especies (Vega y Maroto 1984, García 1997). Muchas plagas se han adaptado y presentan resistencia hacia uno o varios plaguicidas, lo que ha obligado al agricultor a recurrir a la combinación de estos para preparar "cocteles", sin conocer sus implicaciones en la salud humana o para el ambiente (García 1997, Hayes 1991).

A nivel mundial existe desconocimiento acerca del daño que muchas de estas sustancias y sus mezclas pueden ocasionar. Según la
Academia Nacional de Ciencias de los EE.UU., la información toxicológica es suficiente solo para $10 \%$ de los ingredientes activos comercializados de plaguicidas, para $52 \%$ es incompleta y para los $38 \%$ restantes no hay información (García 1997). Si se buscan datos toxicológicos en seres humanos la información se reduce aún más, ya que por razones de tipo ético el estudio de los efectos adversos se realiza generalmente con animales de laboratorio (Castillo et al. 1995).

El uso de plaguicidas es un riesgo para la salud de las comunidades en contacto con ellos, ya sea a mediano, corto o largo plazo. Por eso es tan importante en salud ocupacional 
y preventiva detectar de alguna forma sus efectos en la salud humana, ojalá antes de que estos sean irreversibles (Ashby et al. 1993).

Costa Rica, al igual que el mundo entero, ha tenido que utilizar agroquímicos para poder llevar sus productos al mercado y competir a nivel mundial según las presiones impuestas por los consumidores, pero con el agravante de ser un país pobre, donde la información y los medios de control sobre estas sustancias son deficientes y en donde los estudios de toxicidad en humanos y de contaminación ambiental son escasos cuando no inexistentes (García 1997, Jiménez 1995).

El cultivo del banano aparece como una de las actividades agrícolas donde se producen mayor cantidad de intoxicaciones y problemas de salud en sus trabajadores. La gran vulnerabilidad a las plagas que este cultivo presenta obliga al uso de una gran cantidad de plaguicidas (Wesseling et al. 1993, Quirós et al.1994, Pardo 1996). En Costa Rica, el consumo promedio de plaguicidas por hectárea es tan alto o más alto que en zonas de agricultura intensiva de países desarrollados, llegando a utilizarse hasta $45 \mathrm{~kg}$ de ingrediente activo por hectárea cultivada de banano (Wesseling 1997). Los plaguicidas también pueden causar daños irreversibles a largo plazo en los trabajadores expuestos, un ejemplo histórico es el ocasionado por el nematicida 1,2-dibromo-3-cloropropano (DBCP) utilizado en el cultivo de banano, causante de innumerables afecciones entre las cuales destaca la esterilidad masculina (Ramírez y Ramírez 1980, Vega y Maroto 1984).

Estudios epidemiológicos en poblaciones ocupacionalmente expuestas a plaguicidas (Ashby et al. 1993, Pisani 1994, Wesseling 1997) muestran un aumento en el riesgo para desarrollar diferentes tipos de cáncer, como el de la cavidad oral, la laringe, los pulmones, el esófago, el estómago, el hígado, el páncreas, los riñones y linfomas.

El monitoreo del daño genético en las poblaciones expuestas utilizando biomarcadores se vislumbra como una herramienta útil en la prevención de los tumores. Los micronúcleos constituyen uno de los biomarcadores de efecto utilizados para monitorear el daño genético de poblaciones expuestas, ya sea de células epiteliales o de linfocitos. Son núcleos pequeños que se ubican al lado del núcleo celular que indican algún tipo de aberración cromosómica que pudo producirse como efecto de la exposición a agentes ambientales (Lohman et al. 1984, Tolbert et al. 1991). Las mayores limitaciones que tienen como marcadores de efecto son que no caracterizan la naturaleza del daño nuclear inducido y que existe una considerable variación intra e interindividual (Pisani 1994, Bonassi y Au 2002). El análisis de micronúcleos en células del epitelio oral es un método específico para evaluar efectos clastogénicos (Lohman et al. 1984). Este análisis tiene las siguientes ventajas: las células no necesitan ser cultivadas, los micronúcleos pueden reflejar eventos genotóxicos que han ocurrido en las células de división basal en las últimas tres semanas, la técnica es rápida, simple y las muestras son fáciles de obtener (Tolbert et al. 1991). En algunos estudios se han informado frecuencias más elevadas de micronúcleos en individuos expuestos al consumo de alcohol y tabaco, encontrándose una relación directamente proporcional entre el número de micronúcleos y el tiempo de exposición (Livingston et al. 1990, Stich et al. 1982, Tolbert et al. 1991). Los resultados de otros estudios son variables, dependiendo del tipo de tejido, así como de las condiciones de la investigación en general (Pastor et al. 2001, Pastor et al. 2002).

Existen otras anormalidades nucleares en las células, que también son indicadores de genotoxicidad, y que constituyen muchas veces una limitación de la evaluación de los micronúcleos ya que pueden dificultar el reconocimiento de micronúcleos verdaderos. Estas anormalidades corresponden a diferentes procesos biológicos (Tolbert et al.1991). Las más frecuentes en las células exfoliadas son:

- Células binucleadas: con presencia de dos núcleos dentro de la célula. Probablemente no involucra una interacción directa con el ADN, son producidas a causa de procesos de interferencia que ocurren tarde en la 
división celular (no se conoce bien su origen, ni su efecto en la célula).

- Células con núcleo en "broken eggs" (huevo quebrado): donde el núcleo aparece con una protuberancia de tamaño variable. $\mathrm{Su}$ origen y significado aún se desconoce.

- Células con núcleo picnótico: donde este se observa encogido. En altos niveles es una respuesta de lesión o daño celular.

- Células con núcleo donde la cromatina está condensada: en altos niveles es una respuesta de lesión celular, se distingue de la cariorresis porque la membrana nuclear está intacta.

- Células con núcleo en cariorresis o desintegración nuclear: donde la membrana nuclear desaparece y la cromatina se observa condensada en grupos.

- Células con núcleos en cariolisis o disolución nuclear: donde la membrana nuclear se conserva y la cromatina está en disolución.

Con el fin de determinar el posible daño al material genético por exposición laboral a plaguicidas, en este estudio se utilizó como biomarcador la presencia de micronúcleos en células del epitelio oral de trabajadoras del empaque del banano. Se analizaron también las frecuencias de otras anormalidades en el núcleo de dichas células porque pueden ser indicio de genotoxicidad o de citotoxicidad.

\section{MATERIALES Y MÉTODOS}

Población en estudio: El estudio tuvo un diseño de tipo caso-control. Los casos o mujeres expuestas fueron trabajadoras de plantas empacadoras pertenecientes a fincas bananeras independientes localizadas en Guápiles y Siquirres, región atlántica de Costa Rica, con edades entre 16 y 50 años y que tenían al menos tres meses de laborar en forma continua. El grupo de controles eran mujeres que vivían en la misma región, que nunca habían estado expuestas directamente a plaguicidas en su trabajo, cuyas edades y hábitos eran similares a los del grupo de mujeres expuestas a plaguicidas y que no vivían en fincas bananeras, ni sus esposos o compañeros trabajaban en esta actividad. El tamaño final del grupo de estudio fue de 84 mujeres (40 expuestas y 44 no expuestas). Las mujeres que decidieron colaborar firmaron el consentimiento informado y contestaron un cuestionario sobre información personal, de salud, tipo de anticonceptivo usado, consumo de medicamentos, exposición a rayos $\mathrm{X}$, hábitos de alimentación, cantidad de tazas de café o té negro consumidas al día, historia de exposición laboral, etc. (El cuestionario completo se encuentra en Castro 1999).

Recolección de las muestras: Las muestras biológicas se obtuvieron entre diciembre de 1995 a junio de 1996 y de diciembre de 1996 a junio de 1997, recolectando de cuatro a ocho muestras de epitelio oral por visita a cada planta empacadora. Se obtuvo la muestra haciendo un frotis en la pared interna de ambas mejillas, después de lavar la boca con un enjuague bucal. La mucosa obtenida se puso en solución salina al $0.9 \%$ y se depositó en una hielera aproximadamente a $10^{\circ} \mathrm{C}$, mientras se regresaba al laboratorio, donde se guardaron a $4^{\circ} \mathrm{C}$ hasta el día siguiente, cuando se lavaron con solución salina al $0.9 \%$, se centrifugaron y se fijaron con metanol absoluto.

Micronúcleos del epitelio oral: se realizó mediante la técnica utilizada por Blas (1995).

Análisis de las preparaciones: las preparaciones teñidas se observaron en un microscopio de contraste de fases o uno con el condensador desplazado hasta la posición más inferior. Se contaron mil células por caso, pero si había menos de cinco micronúcleos se contaron mil células más, y si aún así había menos de cinco, se contaron mil más. Para la identificación de los micro núcleos se utilizaron los criterios de Tolbert et al. (1991), y se clasificaron como de alta certeza si contaban con todas las características, pero si le faltaba alguna, se clasificaron como de mediana certeza. También se analizaron las siguientes anormalidades nucleares según la clasificación de Tolbert et al. (1991): células binucleadas, células con núcleo en "broken eggs", células con núcleo picnótico, células 
con núcleo donde la cromatina está condensada, células con núcleo en cariorresis o desintegración nuclear, células con núcleos en cariolisis o disolución nuclear. Se tomó en cuenta los síntomas de genotoxicidad (corresponde a la suma de las células con micronúcleos, las células binucleadas, las células con núcleos "en broken eggs", las células con núcleos picnóticos, las células con núcleos en cariorresis y las células con núcleos en cariolisis), de citotoxicidad (suma de las células con núcleos picnóticos, las células con cromatina condensada, las células con núcleos en cariorresis y las células con núcleos en cariolisis) y de apoptosis (suma de las células con núcleos picnóticos, las células con cromatina condensada y las células con núcleos en cariorresis).

Análisis estadístico: Se realizó usando el paquete de programas estadísticos Statistics para Windows, versión 4.5, 1993. Se compararon todas las variables de la encuesta entre los dos grupos (expuestas y no expuestas), por medio de la estadística $Z$. La información obtenida del análisis de las células de las expuestas y las no expuestas fue transformada a proporciones (número de células observadas con una anormalidad específica entre el número total de células contadas para cada muestra). Para normalizar la distribución de los datos y hacer un manejo adecuado de estos, se utilizó la transformación de arcosenos de la raíz cuadrada (Zar 1996). La información transformada fue sometida a la prueba de normalidad de Kolmogorov-Smirnov. Para comparar la frecuencia de micronúcleos y otras anormalidades del grupo de no expuestas con el grupo de expuestas se usó la prueba t-Student para grupos independientes. Las otras variables (además de la exposición a plaguicidas), elucidadas por el cuestionario, que podrían estar contribuyendo a la aparición de las anormalidades nucleares, se sometieron a un análisis de regresión múltiple (Daniel 1987), el cual ayudó a determinar si existía una relación lineal entre alguna variable Y (cualquier anormalidad nuclear) y las variables $\mathrm{X}$ (cualquier categoría de riesgo), explicativas o de predicción. En el análisis de regresión múltiple, además del coeficiente de determinación $\mathrm{r}^{2}$ (el cual mide la proximidad del ajuste de la ecuación de regresión de la muestra a los valores observados de $\mathrm{Y}$ ); se aplicó también la prueba de hipótesis para beta (coeficiente beta estandarizado), que es una alternativa para probar la hipótesis nula de no relación lineal entre dos variables (la dependiente y la independiente) (Daniel 1987). Finalmente, se aplicó una regresión lineal para detectar si el tiempo de trabajo en una finca bananera y el número de tazas de café que se consume diariamente estaba relacionado con un aumento en la frecuencia de anormalidades nucleares. El nivel de significación aplicado en todas las pruebas estadísticas es de 0.05 .

\section{RESULTADOS}

La edad promedio del grupo de mujeres expuestas es de 33.2 años, que varía entre los 16 y 50 años, con una exposición promedio de 6.4 años. La distribución de edad del grupo de mujeres no expuestas es similar. Sin embargo ambos grupos muestran diferencias importantes en relación a otras variables. Entre las variables o categorías de riesgo que muestran diferencias importantes entre ambos grupos sobresalen la exposición a plaguicidas en el trabajo, el consumo de anticonceptivos orales y dermatitis, todas con porcentajes mucho mayores en el grupo de expuestas, mientras que un alto consumo de café (más de cinco tazas al día) y la presencia de una radiografía dental en el último año antes de tomar la muestra de epitelio oral, muestran porcentajes mayores en el grupo de no expuestas. La prueba de normalidad de Kolmogorov-Smirnof muestra que las variables presentan una distribución normal, con excepción de la proporción de células que poseen núcleos con cromatina condensada. En las variables: células con núcleos en cariorresis, genotoxicidad, citotoxicidad, y apoptosis se observó una diferencia significativa $(\mathrm{p}<0.05)$ entre el grupo de expuestas y no expuestas, presentando mayor número de anormalidades el grupo de mujeres no expuestas. La t-Student que se aplicó para comparar las 
CUADRO 1

Comparación de las medias de diferentes anormalidades en el nucleo de celulas epiteliales, entre el grupo de mujeres expuestas y el grupo de no expuestas a plaguicidas, mediante una prueba t-student

TABLE 1

Comparison of averages of different abnormalities in the nuclei of epithelial cells, between the group of exponed and non exponed women to pesticidas, through the t-student test

$\begin{array}{ll}\text { Variable* } & \text { x casos } \\ & \\ \text { Micronúcleos totales } & 0.0310 \\ \text { Micronúcleos de alta certeza } & 0.0220 \\ \text { Células binucleadas } & 0.0440 \\ \text { Células con núcleos picnoticos } & 0.0310 \\ \text { Células con núcleos en "BE" } & 0.0260 \\ \text { Células con cromatina condensada } & 0.0031 \\ \text { Células con núcleos en cariorresis } & 0.0480 \\ \text { Células con núcleos en cariolisis } & 0.0850 \\ \text { Genotoxicidad } & 0.0940 \\ \text { Citotoxicidad } & 0.1100 \\ \text { Apoptosis } & 0.0630 \\ & \\ \text { * Las variables presentan la transformacion de arcosenos } \\ \text { X = media } \\ \text { G.L. = grados de libertad } \\ \text { N.S.= No significativo con una p }>0.05 . \\ \text { BE = "Brokken eggs" }\end{array}$

medias de micronúcleos de alta certeza en ambos grupos no fue estadísticamente significativa, por lo tanto no existen diferencias significativas entre las medias de ambas muestras (Cuadro 1). Como los resultados obtenidos muestran que las medias de micronúcleos de alta certeza y los micronúcleos totales eran en general muy parecidas, se decidió trabajar solo con las medias de los micronúcleos totales.

Los resultados de la regresión múltiple entre las anormalidades nucleares (cariorresis, genotoxicidad, citotoxicidad y apoptosis) que tienen diferencias estadísticamente significativas mediante la prueba t-Student (Cuadro 1), y los factores que pudieran estar contribuyendo a la aparición de dichas anormalidades se observan en el cuadro 2. Hay una relación directa entre las anormalidades $(\mathrm{p}<0.05)$ y los antecedentes de dermatitis, indicando que las mujeres que sufren de problemas en la piel (que pertenecen en mayoría al grupo de expuestas) presentan una frecuencia mayor de estos tipos de anormalidades. También existe una relación lineal inversa entre estas anormalidades y la exposición a plaguicidas.

A pesar de que la variable cafeína en la prueba de regresión múltiple no mostró una relación lineal con las diferentes variables dependientes, la cantidad de café que se toma diariamente mostró estar relacionada directamente con el aumento en el número de anormalidades nucleares que detectan citotoxicidad (Cuadro 3). El grupo que está más afectado es el de las mujeres no expuestas. También se muestra que existe una relación lineal inversa estadísticamente significativa $(p=0.05)$, entre la citoxicidad y el tiempo de trabajar en la actividad bananera.

\section{DISCUSIÓN}

Según lo establecido por otros estudios (Titenko et al.1994), el promedio normal de las frecuencias de micronúcleos varía considerablemente de una población a otra $(0.03 \%$ a 


\section{CUADRO 2}

Resultados del análisis de regresión múltiple de cada variable dependiente con las variables independientes (explicativas) más influyentes en la posible formación de células anormales

TABLE 2

Multiple analysis regression results of each dependent variable with the independent variables (explanatory) more influential in the possible formation of abnormal cells

\begin{tabular}{|c|c|c|c|c|}
\hline \multirow[t]{2}{*}{ Variables } & \multicolumn{2}{|c|}{$\begin{array}{c}\text { Modelo } 1 \\
\text { Cariorresis } \\
\mathrm{r}^{2}=0.20\end{array}$} & \multicolumn{2}{|c|}{$\begin{array}{l}\text { Modelo } 2 \\
\text { Cariolisis } \\
\mathrm{r}^{2}=0.15\end{array}$} \\
\hline & ß & $\mathrm{T}$ & B & $\mathrm{T}$ \\
\hline Exposición & 0.35 & $\mathrm{p}=0,04$ & 0.20 & N.S. \\
\hline Edad & -0.08 & N.S. & -0.13 & N.S. \\
\hline Fumado & $-0,21$ & N.S. & 0.14 & N.S. \\
\hline Medicamentos & -0.02 & N.S. & 0.10 & N.S. \\
\hline Anticonceptivos & -0.08 & N.S. & 0.08 & N.S. \\
\hline Dermatitis & 0.26 & $\mathrm{p}=0.02$ & 0.22 & $\mathrm{p}=0.05$ \\
\hline Radiografías dentales & 0.03 & N.S. & 0.05 & N.S. \\
\hline Cafeína & 0.01 & N.S. & 0.07 & N.S. \\
\hline Cerveza & -0.12 & N.S. & -0.04 & N.S. \\
\hline Ant. fam. malform. & 0.14 & N.S. & 0.17 & N.S. \\
\hline Problemas para concebir & 0.07 & N.S. & 0.030 & N.S. \\
\hline Hijos con malformaciones & 0.06 & N.S. & -0.06 & N.S. \\
\hline
\end{tabular}

\begin{tabular}{cc} 
Modelo 3 \\
Genotoxicidad \\
\multicolumn{2}{c}{$\mathrm{r}^{2}=0.16$} \\
$\beta$ & $\mathrm{T}$ \\
& \\
0.21 & N.S. \\
-0.07 & N.S. \\
-0.13 & N.S. \\
-0.03 & N.S. \\
0.13 & N.S. \\
0.23 & $\mathrm{p}=0.03$ \\
-0.04 & N.S. \\
-0.07 & N.S. \\
-0.09 & N.S. \\
0.19 & N.S. \\
0.09 & N.S. \\
0.07 & N.S.
\end{tabular}

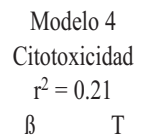

Modelo 5

Apoptosis $\mathrm{r}^{2}=0.19$

\section{$0.24 \mathrm{p}=0.04$}

-0.11 N.S.

-0.17 N.S.

$-0.06 \quad$ N.S.

0.08 N.S.

$0.26 \mathrm{p}=0.02$

0.02 N.S.

$-0.09 \quad$ N.S.

$-0.10 \quad$ N.S.

$0.21 \quad$ N.S.

0.09 N.S.

0.04 N.S.

\section{CUADRO 3}

Comparación del efecto del número de tazas de café y el tiempo de trabajo en una finca bananera sobre la producción de genotoxicidad, citotoxicidad, apoptosis y micronúcleos

TABLE 3

Comparison of the effect in the number of cups of coffee and the working time in a banana land over the production of genotoxicity, citotoxicity, apoptosis and micronucleous

\begin{tabular}{lcccc} 
Variables & Tazas de café(n=81 personas) & \multicolumn{2}{c}{ Tiempo de trabajo (n=40, solo casos) } \\
& $\beta$ & $\mathrm{P}$ & $\beta$ & $\mathrm{p}$ \\
Genotoxicidad & 0.18 & N.S. & -0.28 & N.S. \\
Citotoxicidad & 0.27 & $\mathrm{p}=0.01$ & -0.31 & $\mathrm{p}=0.05$ \\
Apoptosis & 0.17 & N.S. & -0.29 & N.S. \\
Micronúcleos de alta certeza & -0.06 & N.S. & -0.05 & N.S.
\end{tabular}

N.S.= No significativo con una $\mathrm{p} £ 0.05$.

$0.47 \%)$. Estas diferencias dependen de factores genéticos y ambientales tales como la alimentación, la edad y el sexo (Pastor et al. 2002).

Los promedios de las frecuencias de micronúcleos encontradas en este estudio, son de $0.17 \%$ para el grupo de expuestas y de $0.22 \%$ para las no expuestas, lo que se considera dentro de lo normal. Las frecuencias de micronúcleos mayores al límite $(0.47 \%)$ dentro del grupo corresponden a factores individuales de sensibilidad a una o más variables. 
Sin embargo, debido a que en Costa Rica no existen estudios que establezcan un límite normal de promedios de frecuencias para este biomarcador, es mejor limitarse a observar y analizar las variaciones de las frecuencias entre ambos grupos, sin hacer mayor reparo a los límites de normalidad establecidos para otras poblaciones.

La prueba t-Student aplicada para analizar los valores promedio de las frecuencias de micronúcleos de la muestra de expuestas con respecto a las no expuestas, determinó que no existen diferencias significativas entre ambos grupos, esto puede ser consecuencia de una o más de las siguientes alternativas: (1) Que el grupo control no es el adecuado, ya que puede estar expuesto a plaguicidas en diferentes formas; (2) Que el tejido epitelial o/y el biomarcador escogido para el análisis no es el idóneo; (3) Que no existe daño genético a este nivel por exposición a plaguicidas utilizados actualmente en las fincas bananeras.

En un estudio previo realizado en trabajadores de un viñedo en Yugoslavia, se analizaron dos grupos controles, uno constituido por personas de un pueblo aledaño al cultivo y otro por personas cuya residencia estaba alejada $200 \mathrm{~km}$ del viñedo. El grupo control del pueblo aledaño al viñedo tenía aumentada la frecuencia de micronúcleos en células binucleadas de linfocitos. Los investigadores concluyen que dicho aumento se debe a la contaminación, principalmente por aire, que producen los plaguicidas que se aplican en el área (Joksic et al. 1996).

Guápiles y Siquirres pertenecen a una región eminentemente agrícola en donde la población se ha expuesto a plaguicidas durante décadas y en donde una buena parte de los habitantes han trabajado en labores del campo. Esta condición pone en duda que el grupo de no expuestas estuviera a salvo de la exposición a plaguicidas, más aún, un estudio realizado en el Valle de la Estrella pone de manifiesto la posibilidad de contaminación por plaguicidas en aguas subterráneas y superficiales, principalmente por clorotalonil y clorpirifós (Abarca y Ruepert 1992).

Por otro lado, el análisis de niveles de colinesterasa sérica y eritrocítica realizado en un estudio paralelo a este (Cuenca et al. 1997) y con los mismos individuos de esta investigación, encontró altos niveles en la inhibición de las colinesterasas en algunas mujeres del grupo de casos y en algunos controles, fortaleciendo más la idea de que el grupo de controles está expuesto a plaguicidas por el hecho de vivir cerca de las plantaciones.

Lo recomendable sería encontrar otra población que tenga características similares al de las mujeres del grupo de expuestas, pero fuera de una región agrícola, sin embargo, esta condición necesariamente sería urbana, lo que implica exposición a otro tipo de contaminantes, además de las diferencias culturales que determinan cambios en los hábitos de fumado, ingesta de alcohol, etc.

El tejido de los carrillos de la boca es un epitelio escamoso estratificado no queratinizado, muy grueso que protege a los tejidos profundos de la fuerza mecánica de la masticación y forma una barrera poco permeable (Arey y Rea 1968). Las células de las capas superficiales (que corresponden a la muestra analizada) se exponen fácilmente a cualquier sustancia tóxica, sin embargo, la saliva presente en toda la mucosa de la boca protege de sustancias poco solubles en agua, como es el caso de muchos plaguicidas (Berne 1992, Edwards et al. 1991).

Las mujeres que trabajan en las plantas empacadoras se exponen principalmente a los plaguicidas tiabendazol o imazalil, y al insecticida clorpirifós, los cuales presentan baja solubilidad en agua (Worthing 1997). En estos casos, al menos, parece que la mucosa oral se encuentra a salvo.

Los micronúcleos se forman como resultado de fractura cromosómica o por daño a las proteínas que constituyen el huso mitótico (Calvert et al. 1998), cualquier otro tipo de daño al material genético queda fuera del alcance de este biomarcador; por lo tanto, aunque este estudio no pudo determinar daño expresado en forma de micronúcleos en el epitelio oral como consecuencia de la exposición a los plaguicidas, otros estudios confirman que este efecto existe en grupos similares de trabajadoras costarricenses del empaque del banano, cuando se 
utilizan otros biomarcadores (Ramírez y Cuenca 2002, Cuenca y Ramírez 2004). Los estudios de tipo epidemiológico (Pisani 1994, Ashby et al. 1993, Wesseling et al. 1996) han señalado a muchas de estas sustancias como potencialmente carcinógenas, neurotóxicas, teratógenas, mutagénicas, fetotóxicas y de causar efectos negativos en el sistema reproductor de ciertos animales (Hayes 1991), por lo tanto es necesario continuar una estrecha vigilancia sobre la salud de trabajadores expuestos a plaguicidas.

Al agrupar las anormalidades nucleares en tres diferentes categorías: genotoxicidad, citotoxicidad, y apoptosis, se observa que las dos últimas son significativamente más elevadas en las mujeres no expuestas. La genotoxicidad es un proceso que puede llevar a apoptosis (muerte celular programada) y a un aumento en la frecuencia de células con núcleos en cariolisis y en cariorresis puede significar genotoxicidad; por lo tanto, las mujeres que presentaron diferentes tipos de dermatitis también tienen aumentadas las frecuencias de células anormales que conllevan a citotoxicidad, genotoxicidad o ambas. Las mujeres del grupo de casos presentan más problemas de dermatitis que el grupo de controles, y la literatura acumula información de que las personas expuestas a plaguicidas suelen sufrir de problemas en la piel (Cremlyn 1978, Hayes 1991, Vaquerano 1995, García 1997).

El análisis de regresión lineal señaló que el número de tazas de café que suelen tomar diariamente las mujeres de la muestra, está relacionado con el aumento en las frecuencias de anormalidades nucleares que denotan citotoxicidad y apoptosis. Un $11.37 \%$ de las mujeres no expuestas toman 5 o más tazas de café diariamente, mientras que ninguna mujer del grupo de expuestas toma esa cantidad durante el día. Esta diferencia en la cantidad de tazas de café consumida por día es lo que puede estar ocasionando un número de anormalidades nucleares significativamente mayor en las mujeres no expuestas.

Existen numerosos estudios (la mayoría de ellos en mamíferos y en cultivos de células humanas) que han investigado el potencial nutricional y toxicológico del café, en donde se informa de un aumento en la frecuencia de anormalidades nucleares, así como en el intercambio de material genético en cromátidas hermanas de células linfocíticas, en los consumidores de esta bebida (Stadler et al 1994, Anónimo 1997, Wijewickreme y Kitts 1998).

La citotoxicidad y mutagenicidad del café, se debe a la acción oxidativa del radical hidroxilo sobre el ADN que ocasiona la oxidación de las bases nitrogenadas produciendo $\mathrm{N}$-óxido adenina y 8-oxo-2'-deoxiguanosina. Esto da como resultado la pérdida de bases nitrogenadas (Wijewickreme y Kitts 1998).

La habilidad de componentes fenólicos de actuar como anti-oxidantes o como promotores de oxidación depende de la concentración de los otros componentes implicados. Los resultados obtenidos en la investigación realizada por Stadler et al. (1994) revelaron que los bajos niveles de café inhiben la peroxidación lipídica y actúan como anti-oxidante in vitro, mientras que altos niveles de café pueden actuar de una $\mathrm{u}$ otra forma dependiendo del modelo usado (tipo de café, temperatura del agua, concentración presente de otros componentes, etc.).

Se encontró una relación inversa entre el tiempo de trabajo que tiene una mujer en una finca bananera y la frecuencia de células picnóticas y de anormalidades que denotan citotoxicidad. Esta relación refleja que en muchos casos las mujeres con menos tiempo de trabajar en esta actividad deben trabajar también en el campo donde se exponen a todos los plaguicidas, mientras que las más antiguas realizan trabajos (especializados) principalmente o exclusivamente en la planta empacadora. Es importante que en estudios futuros se tome en cuenta este detalle.

Existen mecanismos de reparación y de eliminación de células anormales que todavía no se conocen bien, por ejemplo Calvert et al. (1998), mencionan que la evidencia sugiere que la detección de mutaciones en el gen hprt, depende del intervalo de tiempo transcurrido entre el momento de la exposición y la detección, así como de la intensidad. El hprt es un gene en el cromosoma $\mathrm{X}$ que codifica una 
enzima involucrada en la regulación de la síntesis de las purinas, puede sufrir mutaciones tipo sustituciones o pérdidas de bases nitrogenadas. Estos investigadores encontraron que las exposiciones recientes eran más predictivas (encontraron mayor número de mutaciones en hprt) que las exposiciones crónicas pasadas. La explicación que surge es que las células mutantes son selectivamente removidas cuando la exposición es crónica.

Un estudio de genotoxicidad en trabajadores expuestos a bromuro de metilo, realizado por Calvert et al. (1998), señala que puede existir un comportamiento a nivel celular, que implique falta de respuesta a una exposición o respuesta disminuida. Este tipo de comportamiento se ha observado en células epiteliales de la bucofaringe expuestas a un agente tóxico y usando como biomarcador a los micronúcleos, donde la ausencia de respuesta puede deberse a que el agente genotóxico en cuestión, produce una disminución en la proliferación celular (se necesita división celular para producir micronúcleos) o un aumento en la citotoxicidad.

Mecanismos similares a estos podrían explicar la relación inversa entre el tiempo de trabajo en una finca bananera que tiene una mujer y la frecuencia de células picnóticas y de anormalidades que denotan citotoxicidad.

\section{AGRADECIMIENTOS}

A las trabajadoras del empaque de banano que generosamente colaboraron con esta investigación, a Jorge Lobo Segura, por su orientación en la aplicación de las pruebas estadísticas, al personal del INISA, en especial a Federico Hernández Villalobos, a Jaime García González, por su apoyo durante toda la ejecución de este proyecto, al proyecto PLAGSALUD/OPS, al Servicio Alemán de Intercambio Académico (DAAD), a la Vicerrectoría de Investigación de la Universidad de Costa Rica (proyecto $\mathrm{N}^{\circ} 742-95-277$ ).

\section{RESUMEN}

Para determinar si la exposición laboral a plaguicidas produce daño al material genético se utilizó como biomarcador de efecto la presencia de micronúcleos en células del epitelio oral de trabajadoras de Guápiles y Siquirres, Costa Rica. También se analizaron otras anormalidades nucleares que pueden ser indicio de genotoxicidad o de citotoxicidad. El grupo de mujeres expuestas a plaguicidas trabajaban en plantas empacadoras de diferentes fincas bananeras independientes. El grupo de mujeres no expuestas nunca habían trabajado en labores agrícolas, no vivían dentro de una finca bananera, así como tampoco sus esposos o compañeros. Se obtuvo información acerca del estilo de vida, historia médica y familiar de las participantes mediante una entrevista. No se encontró un aumento significativo en la frecuencia de micronúcleos en el grupo de expuestas con respecto a las no expuestas. Las otras anormalidades nucleares mostraron indicios de citotoxicidad y genotoxicidad en el grupo de no expuestas, asociados a ingesta de café y a radiografías dentales. Estos resultados no descartan a los plaguicidas como agentes capaces de causar daño genético, sino que más bien los micronúcleos de la mucosa oral no parecen ser el biomarcador más adecuado para medirlo.

\section{REFERENCIAS}

Abarca, L. \& C. Ruepert.1992. Plaguicidas encontrados en el Valle de la Estrella: Estudio preliminar. Tecnología en Marcha. 12(3): 31-38.

Ashby, J., W. Anwar, W.W. Au, A. Massoud \& J. M. Gentile. 1993. Genetic toxicology in developing countries: comments and recommendations. Environ. Health Perspect. Suppl. 101(3): 335-338.

Arey, L. \& R.L. Rea. 1968. Histología humana. Traducido del inglés por el Dr. José Perea Sasiain. Fournier. México, D.F. 379 p.

Berne, R. \& M. Levi. 1992. Principles of physiology. Mosby/ Doyma. Barcelona, 680 p.

Blas, J.T. 1995. Evaluación de la frecuencia de micronúcleos en células uroepiteliales de individuos con exposición ambiental a arsénico. Tesis de Licenciatura. Universidad Autónoma de México, Facultad de Ciencias. México, D.F. 64 p.

Bonassi, S. \& W. Au. 2002. Biomarkers in molecular epidemiology studies for health risk prediction. Mut. Res. 511: 73-86. 
Calvert, G., G.Talaska, C.Müller, M.Ammenheuser, W. Au, J. Fajen, L. Fleming, T. Briggle \& E. Ward.1998. Genotoxicity in workers exposed to methyl bromide. Mutat. Res. 417: 115-128.

Castillo, L., F. Chaverri, C. Ruepert \& C. Wesseling. 1995. Manual de plaguicidas. Guía para América Central. Programa de Plaguicidas: Desarrollo, Salud y Ambiente. EUNA. San José. 680 p.

Castro, R. 1999. Análisis de micronúcleos de epitelio oral en trabajadoras de una zona bananera expuestas a plaguicidas. Tesis de Licenciatura en biología con énfasis en genética humana. Universidad de Costa Rica. San José, Costa Rica. 101 p.

Cremlyn, R. 1978. Plaguicidas modernos y su acción bioquímica. Traducción del inglés por Esther Baradon De Frixione y Eugenio Frixione. Limusa. México, DF. 355 p.

Cuenca, P., V. Ramírez \& R. Castro. 1997. Efecto genotóxico de los plaguicidas en una población costarricense expuesta ocupacionalmente. Informe final al Proyecto PLAGSALUD. Sección de Genética Humana, INISA y Escuela de Biología. Universidad de Costa Rica. 47 p.

Cuenca, P., V. Ramírez. 2004. Aberraciones cromosómicas en trabajadoras expuestas a plaguicidas. Rev. Biol. Trop. 52: 219-224.

Daniel, W. W. 1987. Bioestadística. Traducido del inglés por Manuel Guzman Ortiz. Noriega. México D.F. $667 \mathrm{p}$.

Edwards, R., D.H. Ferry \& W.A. Temple. 1991. Fungicides and related compounds, pp. 1409-1470. In Hayes, W. J. \& E. R. Laws (eds.). Handbook of pesticide toxicology. Vol. 3. Classes of pesticides. Academic, San Diego.

García, J. E. 1997. Introducción a los plaguicidas. EUNED. San José. Costa Rica, 450 p.

Hayes,W.J. 1991. Pesticide problems and their solutions, pp. 27-29. In Hayes, W. J. and E.R. Laws (eds). Handbook of pesticide toxicology. Vol 1. General principles. Academic, San Diego.

Jiménez, J. 1995. Plaguicidas y salud en las bananeras de Costa Rica. ASEPROLA (Asociación de Sercicios de Promoción Laboral). San José. Costa Rica. $144 \mathrm{p}$.

Joksic, G., A. Vidakovic \& V. Spasojevic-Tisma. 1996. Cytogenetic monitoring of pesticide sprayers. Environ. Res. 75: 113-118.
Livingston, G., R.N. Reed, B.L. Olson \& J.E. Lockey. 1990. Induction of nuclear aberrations by smokeless tobacco in epithelial cells of human oral mucosa. Environ. Mol. Mutag. 15: 136-144.

Lohman, P. H. M., J. D.Jansen \& R. A. Baan. 1984. Comparison of various methodologies with respect to specificity and sensitivity in biomonitoring occupational exposure to mutagens and carcinogens. pp. 259-277. In Berlin A., M. Draper, K. Hemminki \& H. Vainio. (eds.). Monitoring human exposure to carcinogenic and mutagenic agents. IARC. Scientific Publications. No. 59. Lyon. Francia.

Pardo, J. 1996. El cultivo del banano. EUNED. San José. 73 p.

Pastor, S., S. Gutiérrez, A. Creus, N. Xamena, S. Piperakis \& R.Marcos. 2001. Cytogenetic análisis of Greeks farmers using the micronucleous assay in peripheral lymphocytes and bucal cells. Mutagenesis. 16: $539-545$

Pastor, S., A. Creus, N. Xamena, C. Siffel \& R. Marcos. 2002. Occupational exposure to pesticidas and cytogenetic damage: Results of a hungarian population study using the

micronucleus assay in lymphocytes and bucal cells. Environ. Mol. Mutagen. 40: 101-109.

Pisani, P. 1994. Burden of cancer in developing countries. pp. 31-39. In N.P earce, E. Matos, H. Vainio., P. Boffetta \& M. Kogevinas (eds.). Occupational cancer in developing countries. IARC. Scientific Publications. No. 129. Lyon.

Quirós, D., A. Salas \& Y. Leveridge. 1994. Intoxicaciones con plaguicidas en Costa Rica. Centro Nacional de Intoxicaciones Hospital Nacional de Niños. Editorial Nacional de Salud y Seguridad Social. San José. pp. 7-33.

Ramírez, A.L. \& C. Ramírez. 1980. Esterilidad masculina causada por la exposición laboral al nematicida 1,2-Dibromo-3 Cloropropano. Act. Méd. Cost. 23: 219-222.

Ramírez, V. \& P. Cuenca. 2002. Daño al ADN en trabajadoras bananeras expuestas a plaguicidas en Limón, Costa Rica. Rev. Biol. Trop. 50: 507-518.

Stadler, R., R. Turesky, O. Müller, J. Markovic \& P. Leong-Morgenthaler. 1994. The inhibitory effects of coffe on radical-mediated oxidation and mutagenicity. Mut. Res. 308: 177-190.

Stich, H. F., R. Curtis \& B.B. Parida. 1982. Application of the micronucleos test to exfoliated cells of high cancer risk groups: tobacco chewers. Int. J. Cancer. 30: $553-550$ 
Titenko-Holland, N., L.E. Moore \& M.T. Smith. 1994. Measurement and characterization of micronuclei en exfoliated human cells by fluorescence in situ hybridization with a centromeric probe. Mut. Res. 312: 39-50.

Tolbert, P .E., C.M. Shy \& J.W. Allen. 1991. Micronuclei and other nuclear anomalies in buccal smears a field test in snuff users. Am. J. Epidemiol. 134: 840-850.

Vaquerano, B. 1995. Caracterización de la exposición dermal ocupacional a plaguicidas en una finca bananera en Costa Rica. Tesis de maestría en Salud Pública. Universidad de Costa Rica. San José. 75 p.

Vega, S. \& I. Maroto.1984. Plaguicidas de uso restringido en Estados Unidos se importan libremente en Costa Rica. Rev. Ciencias Amb., Costa Rica. 5-6: 125-134.

Wesseling, C. 1997. Health effects from pesticide use in Costa Rica an epidemiologic approach. Tesis de doctorado. Karolinska Institute. Stockholm. 308 p.

Wesseling, C., L. Castillo \& C. Elinder.1993. Pesticide poisons in Costa Rica. Scand. J. Work Environ. Health. 19: 227-235.
Wesseling, C., A. Ahlbom, D. Antich, A. Rodríguez \& R.Castro.1996. Cancer in banana plantation workers in Costa Rica. Int. J. Epid. Assoc. 25: 1125-1131.

Wijewickreme, A. \& D. Kitts.1998. Modulation of metalinduced cytotoxicity by maillard reaction products isolated from coffee brew. J. Toxicol. Environ. Health. 55: 151-168.

Worthing, C. 1997. The pesticides manual. Crops research Institute. Glasshouse british Crop Protection Council. London, England. 655 p.

Zar, J. 1996. Biostatistical analysis. Prentice Hall. New Jersey, 736 p.

\section{REFERENCIAS EN INTERNET}

Anónimo.1997. COFFEE (group 2B). Summary of data reported and evaluation. International Agency of Research in Cancer (IARC). Lyon, Francia. (Consultada el 14 de julio de 2003, http://193.51,164.11/ htdocs/Monographs/Vol51/01-Coffee.htm). 